\author{
Jia-Yush Yen \\ Professor. \\ Shih-Jung Huang \\ Ph.D. \\ Shu-Shong Lu \\ Department of Mechanical Engineering, \\ National Taiwan University, \\ Taipei, Taiwan, 10617 \\ e-mail: jyen@ccms.ntu.edu.tw
}

\section{A New Compensator for Servo Systems With Position Dependent Friction}

In this study, we present a new compensator design for systems with position dependent friction. Position dependent friction occurs between revolving parts that are conventionally used in mechanical systems. Because the friction force changes with the position, the conventional servo controller can not maintain consistent performance. In this paper, a modified Karnopp function is proposed to more accurately describe the friction force's position dependency. A control methodology then exploits this position dependent friction model in order to cancel out the friction force variation and achieve ultimate boundedness [18]. Experimental results demonstrate that the controller achieves consistently good performance. Based on these results, a compensator design procedure is provided.

\section{Introduction}

This paper addresses the control problem for systems with position dependent friction. Position dependent friction often occurs in high precision systems in which transmission mechanisms are commonly used to achieve high-resolution movements. Transmission mechanism can be in the form of worm gears, rack and pinions, or just conventional transmission boxes. These mechanisms experience rotational contact among various parts. Thus, they all suffer from a certain degrees of position dependent variation in the frictional forces. Friction compensation is used to estimate the friction force and to inject an opposite force to cancel its effect [1-5]. Many friction force models are available for use in friction estimation [6-7]. Such models include the Coulomb friction model [6, 7], the Karnopp model [8], the integral-reset model [9], and the bristle-model [9]. Some of these models can accurately predict the stick-slip behavior $[8,9]$; in addition, some of these models examine the micro-scale contact phenomenon to establish very fine force variations $[9,10]$. Using these models, the controller will calculate the friction force and cancel it in the feed-forward loop; some feedback actions will then compensate for the unmodeled variations.

The control fails in the case where the position dependent friction variation is large. In this case, the controller may be compensating in the wrong direction and thereby lead to an unstable system. From the control perspective, the friction models focus on the friction force's position independent behavior. Therefore, they can adequately regulate the fixed-point position. The controller can always be tuned for a fixed level of friction force; however, when the friction force changes with the position, the controller will fail. This condition becomes especially serious in the case of integral control. The control effort may build up too fast while the friction force decreases with the position. Many research efforts have focused on applying the adaptive control to friction compensation [13-15]. This approach also provides good results only for the position independent friction force. For position dependent friction, the adaptation rate is too slow, thereby leading to system instability. The control problem for position dependent friction is so complicated that some reports have actually claimed that control cannot be accomplished [5].

In this study, we propose a modified Karnopp model. The model assumes that position dependent information is prescribed. As

Contributed by the Dynamic Systems and Control Division for publication in the Journal of Dynamic Systems, MEAsurement, AND Control. Manuscript received by the Dynamic Systems and Control Division April 18, 1996. Associate Technical Editor: Tsu-Chin-Tsao. mentioned above, conventional controllers do not function properly in the systems with position dependent friction because they are adjusted for a relatively limited range of operation [16]. Based on the modified Karnopp model, an extension to the robust controller in [18] is proposed. The controller performs velocity estimation to obtain feedback information and then uses an integral type feedback to eliminate the friction effect. A stability analysis shows that the controller achieves uniformly ultimate boundedness control. From the integral action, it is also insensitive to friction level drifting in the disturbance force, and can effectively cancel out its effect. A design procedure is then provided for the proposed controller design. The analytical results and experimental results demonstrate the effectiveness of the controller performance.

\section{System Description}

Consider a first-order servo system with position-dependent stick-slip friction:

$$
\begin{gathered}
\nu_{r}=\dot{\theta}, \\
\dot{\nu}_{r}=-a \nu_{r}+b\left[\tau-\tau_{f}\left(\theta, \nu_{r}, \tau\right)\right],
\end{gathered}
$$

where $a$ and $b$ are the system parameters, $\theta$ is the angular position, $\nu_{r}$ is the angular velocity, $\tau$ is the applied input, and $\tau_{f}\left(\theta, \nu_{r}, \tau\right)$ represents the friction effect.

The friction model, $\tau_{f}\left(\theta, \nu_{r}, \tau\right)$, includes a position-dependent effect and a stick-slip effect. It is assumed that these two effects can be separated. Therefore, the friction force, $\tau_{f}\left(\theta, \nu_{r}, \tau\right)$, is described by a modified Karnopp model:

$$
\tau_{f}\left(\theta, \nu_{r}, \tau\right)=\tau_{\mathrm{pos}}(\theta) \tau_{\mathrm{kar}}\left(\nu_{r}, \tau\right)
$$

where $\tau_{\text {pos }}(\theta)$ not only represents the magnitude of the stick saturation force with respect to position, but also satisfies a global Lipschitz condition. Moreover, $\tau_{\mathrm{kar}}\left(\nu_{r}, \tau\right)$ represents the factor of the stick-slip force. Thus, $\left\|\tau_{\mathrm{kar}}\left(v_{r}, \tau\right)\right\|$ is no greater than $1, \forall \nu_{r}$, and $\tau$. A more detailed description of the friction model is provided later.

Consider that $\tau_{\mathrm{pos}}(\theta)$ is a position-dependent periodic friction function which can be represented by the summation of sine functions varying with the position. Therefore, the period and amplitude and phase are parameters of position-dependent friction. The stick model can be given by

$$
\tau_{\text {pos }}(\theta)=a_{0}+\sum_{n} a_{n} \cos \left(\frac{2 \pi}{T_{n}} \theta+\alpha_{n}\right),
$$




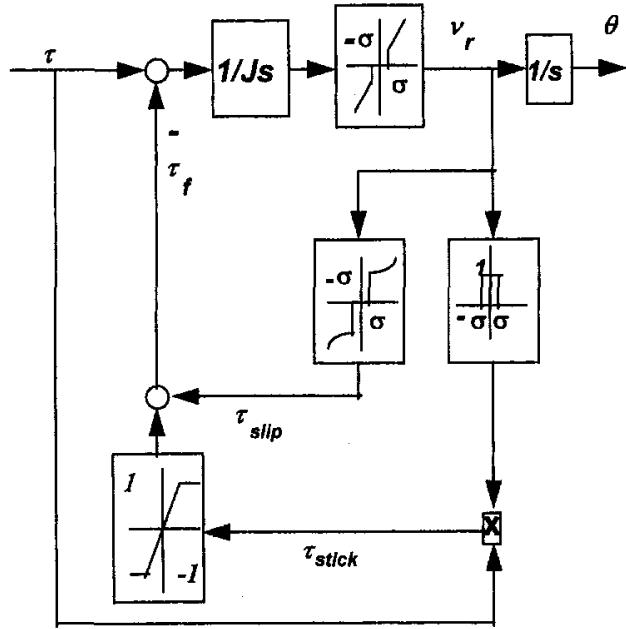

Fig. 1 Modified Karnopp model

where $a_{0}$ represents the average; $a_{n}$ represents the amplitude; $T_{n}$ represents the period and $\alpha_{n}$ represents the phase. In the case studied here, $\tau_{\text {pos }}(\theta)$ is assumed to be a continuously differentiable function and to satisfy a global Lipschitz condition.

Position-dependent friction is not easy to compensate [5]. While its period and amplitude can be determined precisely, its phase is not constant. In fact, the phase varies not only between motions differing in velocity, but also motions of equal velocity. Moreover, the variation was not small but is as much as the period of the function. Using a compensation function of proper amplitude and frequency to reduce the effect of this friction just made matters worse, as the compensation function and actual friction are out of phase. In addition, variation of the phase is possibly the result of backlash in the gear drive, such that the relative physical orientations of the gears are different at the start of each movement.

2.1 Modified Karnopp Model. In the friction model (2), the stick-slip factor $\tau_{\text {kar }}\left(\nu_{r}, \tau\right)$ was modified by Karnopp [8], as indicated in Fig. 1. In the modified Karnopp model, $J$ is the inertia; $\tau_{\text {slip }}$ and $\tau_{\text {stick }}$ denote the slip and stick frictional factors; and $\sigma$ is the limiting angular velocity in the stick and slip regions.

The modified Karnopp model is given by

$$
\tau_{\mathrm{kar}}\left(\nu_{r}, \tau\right)=\tau_{\text {slip }}\left(\nu_{r}\right)\left[\lambda\left(\nu_{r}\right)\right]+\tau_{\text {stick }}(\tau)\left[1-\lambda\left(\nu_{r}\right)\right]
$$

and

$$
\lambda\left(\nu_{r}\right)= \begin{cases}1, & \text { for }\left|\nu_{r}\right|>\sigma \\ 0, & \text { for }\left|\nu_{r}\right| \leq \sigma, \quad \sigma>0\end{cases}
$$

The stick friction factor is represented by

$$
\tau_{\text {stick }}(\tau)= \begin{cases}1, & \text { for } 0<\tau_{\text {pos }}(\theta) \leq \tau \\ \tau / \tau_{\text {pos }}, & \text { for }-\tau_{\text {pos }}(\theta)<\tau \leq \tau_{\text {pos }}(\theta) \\ -1, & \text { for } \tau<-\tau_{\text {pos }}(\theta)<0\end{cases}
$$

where $\tau_{\text {pos }}(\theta)$ is the stick saturation friction and $\tau_{\text {stick }}(\tau)$ represents the stick factor of the friction when $\left|\nu_{r}\right| \leq \sigma .1$ and -1 gives the positive and negative limits of the stick factor. When $\left|\nu_{r}\right| \leq \sigma$, the magnitude of the applied force is not larger than the limit on the stick force; in addition, the friction is equal to the applied force, and the stick factor $\tau_{\text {stick }}(\tau)$ is equal to $\tau / \tau_{\text {pos }}(\theta)$. Moreover, the value of $\sigma$ is set to a small positive number in the simulation to ensure that the numerical integration algorithms remain stable.

The slip friction factor is modeled by

$$
\tau_{\text {slip }}\left(\nu_{r}\right)=\tau_{d}^{+}\left(\nu_{r}\right) U\left(\nu_{r}-\sigma\right)+\tau_{d}^{-}\left(\nu_{r}\right) U\left(-\nu_{r}-\sigma\right) .
$$

$U$ in (7) is the step function. The function $\tau_{d}^{+}\left(\nu_{r}\right)$ is the slip friction factor for a positive velocity, and the function $\tau_{d}^{-}\left(\nu_{r}\right)$ is the slip friction factor for a negative velocity. The magnitudes of $\tau_{d}^{+}\left(\nu_{r}\right)$ and $\tau_{d}^{-}\left(\nu_{r}\right)$ are not presumed to be symmetric. Generally speaking, the magnitudes of $\tau_{d}^{+}\left(\nu_{r}\right)$ and $\tau_{d}^{-}\left(\nu_{r}\right)$ are no greater than 1 .

The model uses the position dependent force variation only in the stick friction portion because the stick friction force dominates the high precision positioning process. Equation (2) is used in the controller design of $\tau_{f}\left(\theta, \nu_{r}, \tau\right)$ as well as in the stability analysis.

\section{Controller Design}

The servo-controller design has two main objectives. First, a controller must be designed that guarantees system stability thereby assuring that the system trajectories are ultimately bounded in a sufficiently small neighborhood of the origin. Consequently, the steady-state specifications and precision specification for the closed-loop system will be satisfied. Second, a controller should be designed that guarantees satisfaction of the transient specifications. In an index system, overshoot and risetime are of primary concern.

Therefore, the controller design process described in this paper will proceed in two stages. The first stage, it will be demonstrated that the proposed controller achieves ultimate boundedness. In the second stage, the detailed parameter design will be presented. We will show how to adjust the design parameters to improve system performance, so that the desired specifications will be satisfied.

Define the angular position $\theta=x_{2}$, the angular velocity $\nu_{r}=$ $x_{3}$, and introduce an augmented state $x_{1}=\int \theta d t y=\theta$ is the system output; $\tau$ is the system input; and $\tau_{f}$ is defined in (2). The nonlinear system in (1) is described by a state equation of the form

$$
\begin{gathered}
\dot{\mathbf{x}}=\mathbf{A} \mathbf{x}+\mathbf{B} \tau-\mathbf{B} \tau_{f}(\mathbf{x}, \tau), \\
y=\mathbf{C} \mathbf{x},
\end{gathered}
$$

where $\mathbf{x}=\left[\begin{array}{lll}x_{1} & x_{2} & x_{3}\end{array}\right]^{T}$ represents is the system states:

$$
\mathbf{A}=\left[\begin{array}{ccc}
0 & 1 & 0 \\
0 & 0 & 1 \\
0 & 0 & -a
\end{array}\right], \quad \mathbf{B}=\left[\begin{array}{l}
0 \\
0 \\
b
\end{array}\right], \quad \mathbf{C}=\left[\begin{array}{lll}
0 & 1 & 0
\end{array}\right]
$$

Before describing the controller design procedure, some equations to be used in this section are provided. Considering the system in (8), the pair $(\mathbf{A}, \mathbf{B})$ is controllable. Therefore, an appropriate state feedback $\mathbf{K}$ can be found such that matrix $\mathbf{A}_{\mathbf{K}}=\mathbf{A}+\mathbf{B K}$ is a Hurwitz matrix. From the Lyapunov stability theorem, there exists a symmetric positive definite matrix $Q \in \mathfrak{R}^{3 \times 3}$, such that the Lyapunov Matrix Equation

$$
\mathbf{A}_{\mathbf{K}}^{T} \mathbf{P}+\mathbf{P A}_{\mathbf{K}}=-\mathbf{Q}
$$

has a corresponding unique solution for $\mathbf{P}$, and $\mathbf{P}$ is positive definite.

The controller design is an extension to the robust controller in [18] with

$$
u=\mathbf{K} \mathbf{x}+\hat{\tau}_{\mathrm{kar}} \int \frac{d \hat{\tau}_{\mathrm{pos}}\left(x_{2}\right)}{d x_{2}} x_{3} d t+\phi(\mathbf{x}),
$$

where $\mathbf{K}$ represents state feedback gains for $\mathbf{A}_{\mathbf{K}}$ to be Hurwitz, and $\hat{\tau}_{\text {kar }}$ and $\hat{\tau}_{\text {pos }}$ are estimated from experimental data. $x_{3}$ is the system velocity. $\left\|\hat{\tau}_{\text {karr }}\left(x_{3}, \tau\right)\right\| \leq 1, \forall x_{3}$ and $\tau$, and $\hat{\tau}_{\text {pos }}\left(x_{2}\right)$ is a continuously differentiable function which satisfies a global Lipschitz condition, and

$$
\phi(\mathbf{x})=-\gamma(\mathbf{x}) \mathbf{B}^{T} \mathbf{P} \mathbf{x}
$$

Notice that $x_{3}$ in (10) can come form direct measurement. Most servomotors are equipped with position encoders, which allow very good estimations for the values of $d \hat{\tau}_{\text {pos }}\left(x_{2}\right) / d x_{2}$. The introduction of the derivative term cancels the variation portion of the 
position dependent friction. The less predictable bias force will than be treated with the nonlinear feedback term $\phi(x)$ in conjunction with the integral action. The resulting control thus constitutes the structure of a robust controller in [18].

From (9), $\mathbf{P}$ is the positive definite solution and the scalar function $\gamma(\mathbf{x})$ is given by

$$
\gamma(\mathbf{x})=\frac{k_{1}\|\mathbf{x}\|+k_{2}}{\left\|\mathbf{B}^{T} \mathbf{P x}\right\|+\epsilon}
$$

with $k_{1}, k_{2} \geq 0$, and $\epsilon>0$. This design term $\phi(\mathbf{x})$ is used to overcome the uncertain effect that is caused by friction uncertainty.

The system in (8) subject to (10) with (11) is given by

$$
\dot{\mathbf{x}}=\mathbf{A}_{K} \mathbf{x}-\gamma(\mathbf{x}) \mathbf{B B}{ }^{T} \mathbf{P} \mathbf{x}+\mathbf{B} d(\mathbf{x})
$$

where $d(\mathbf{x})$ is given by

$$
\begin{aligned}
d(\mathbf{x}) & =\hat{\tau}_{\mathrm{kar}} \int \frac{d \hat{\tau}_{\mathrm{pas}}\left(x_{2}\right)}{d x_{2}} x_{3} d t-\tau_{f} \\
& =\hat{\tau}_{\mathrm{kar}} \int \frac{d \hat{\tau}_{\mathrm{pos}}\left(x_{2}\right)}{d x_{2}} \frac{d x_{2}}{d t} d t-\tau_{f} \\
& =\hat{\tau}_{\mathrm{kar}}\left(\hat{\tau}_{\mathrm{pos}}-c\right)-\tau_{f} \\
& =\hat{\tau}_{f}-c \hat{\tau}_{\mathrm{kar}}-\tau_{f}
\end{aligned}
$$

with $\hat{\tau}_{f}\left(x_{2}, x_{3}, \tau\right)$ represents $\hat{\tau}_{\text {kat }} \hat{\tau}_{\text {pos }}$, and $c$ is a constant. From (4), $\left\|\tau_{\mathrm{kar}}\left(x_{3}, \tau\right)\right\|$ and $\left\|\hat{\tau}_{\mathrm{kar}}\left(x_{3}, \tau\right)\right\|$ are not greater than 1. Therefore,

$$
\begin{aligned}
\|d(\mathbf{x})\| & \leq\left\|\hat{\tau}_{f}-\tau_{f}\right\|+\|c\|\left\|\hat{\tau}_{\text {kar }}\right\| \\
& \leq\left\|\hat{\tau}_{\text {pos }}\right\|\left\|\hat{\tau}_{\text {kar }}\right\|+\left\|\tau_{\text {pos }} \mid\right\|\left\|\tau_{\text {kar }}\right\|+\|c\|, \\
& \leq\left\|\hat{\tau}_{\text {pos }}\right\|+\left\|\tau_{\text {pos }}\right\|+\|c\|
\end{aligned}
$$

Moreover, $\tau_{\text {pos }}\left(x_{2}\right)$ and $\hat{\tau}_{\text {pos }}\left(x_{2}\right)$ satisfy a global Lipschitz condi tion. Therefore, there exist finite positive constants $d_{1}$ and $d_{2}$, such that

$$
\|d(\mathbf{x})\| \leq d_{1}\|\mathbf{x}\|+d_{2}, \quad \forall \mathbf{x} \in \Re^{3} .
$$

Consider the ellipsoid $E(k) \equiv\left\{\mathbf{x} \in R^{n} \mid \mathbf{x}^{T} \mathbf{P} \mathbf{x} \leq k=\right.$ constant $>$ $0\}$, one can define $\underline{E}(\underline{k})$ as the smallest ellipsoid which contains the ball $B(\eta)$, where $\underline{k}=\lambda_{\max }(\mathbf{P}) \eta^{2}$. The ellipsoid $E(\bar{k})$ is the ellipsoid with $\bar{k}>\underline{k}$, and $E\left(k_{0}\right)$ is the ellipsoid with $k_{0}=\mathbf{x}_{0}^{T} \mathbf{P} \mathbf{x}_{0}$.

Definition [18] Uniformly boundedness: Given a solution $\mathbf{x}(\cdot)$ : $\left[t_{0}, t_{1}\right] \rightarrow R^{n}, \mathbf{x}\left(t_{0}\right)=\mathbf{x}_{0}$, say of (10) and (13), it is uniformly bounded if there is a positive constant $\xi\left(\mathbf{x}_{0}\right)<\infty$, possibly dependent on $\mathbf{x}_{0}$ but not on $t_{0}$, such that $\|\mathbf{x}(t)\| \leq \xi\left(\mathbf{x}_{0}\right)$ for all $t \in\left[t_{0}, t_{1}\right]$.

Definition [18] Uniformly ultimate boundedness: Given a solution $\mathbf{x}(\cdot):\left[t_{0}, \infty\right) \rightarrow R^{n}, \mathbf{x}\left(t_{0}\right)=\mathbf{x}_{0}$, it is uniformly ultimately bounded with respect to set $S$ if there is a non-negative constant $T\left(\mathbf{x}_{0}, S\right)<\infty$, possibly dependent on $\mathbf{x}_{0}$ and $S$ but not on $t_{0}$, such that $\mathbf{x}(t) \in S$ for all $t \geq t_{0}+T\left(\mathbf{x}_{0}, S\right)$.

Theorem. The trajectories of the system in (8) subject to controller (10) with (9), and with $k_{1} \geq d_{1}, k_{2} \geq d_{2}$ is uniformly bounded with $\xi(\mathbf{x})=\eta(\epsilon)$, and is ultimately bounded with respect to $\mathbf{x}(\cdot)$, with

$$
T\left(\mathbf{x}_{0}, E(\bar{k})\right)= \begin{cases}\frac{k_{0}-\bar{k}}{c} & \text { for } \mathbf{x}_{0} \notin E(\bar{k}) \\ 0 & \text { for } \mathbf{x}_{0} \in E(\bar{k})\end{cases}
$$

$$
V(\mathbf{x})=\frac{1}{2} \mathbf{x}^{\top} \mathbf{P} \mathbf{x}
$$

as a Lyapunov function for the closed-loop system. The derivative of $V(\mathbf{x})$ is given by

$\dot{V}(\mathbf{x})=\frac{1}{2} \mathbf{x}^{T}\left(\mathbf{A}_{K}^{T} \mathbf{P}+\mathbf{P} \mathbf{A}_{K}\right) \mathbf{x}$

$$
+\gamma(\mathbf{x}) \mathbf{x}^{T} \mathbf{P B B}{ }^{T} \mathbf{P x}+d(\mathbf{x}) \mathbf{B}^{T} \mathbf{P x} .
$$

Noting that $\left\|\mathbf{B}^{T} \mathbf{P x}\right\|^{2} /\left(\left\|\mathbf{B}^{T} \mathbf{P x}\right\|+\boldsymbol{\epsilon}\right) \geq\left\|\mathbf{B}^{T} P x\right\|-\boldsymbol{\epsilon}$, subject to (9) and (15), we have

$$
\begin{aligned}
\dot{V}(\mathbf{x}) \leq-\frac{1}{2} \mathbf{x}^{T} \mathbf{Q} \mathbf{x}-k_{1}\|\mathbf{x}\|\left\|\mathbf{B}^{T} \mathbf{P} \mathbf{x}\right\|-k_{2}\left\|\mathbf{B}^{T} \mathbf{P} \mathbf{x}\right\| & \\
& +k_{1} \epsilon\|\mathbf{x}\|+k_{2} \epsilon+\|d(\mathbf{x})\|\left\|\mathbf{B}^{T} \mathbf{P} \mathbf{x}\right\| \\
\leq-\frac{1}{2} \mathbf{x}^{T} \mathbf{Q} \mathbf{x}-\left(k_{1}-d_{1}\right)\|\mathbf{x}\|\left\|\mathbf{B}^{T} \mathbf{P x}\right\|-\left(k_{2}-d_{2}\right)\left\|\mathbf{B}^{T} \mathbf{P} \mathbf{x}\right\| & +k_{1} \epsilon\|\mathbf{x}\|+k_{2} \epsilon
\end{aligned}
$$

where $\bar{\sigma}(\cdot)$ denotes the spectral radius.

From (19), there exists $\eta(\epsilon)>0$, such that $\dot{V}(\mathbf{x})<0, \forall\|\mathbf{x}\| \geq$ $\eta(\epsilon)$, where

$$
\eta(\boldsymbol{\epsilon})=\frac{k_{1} \epsilon+\sqrt{k_{1}^{2} \epsilon^{2}+2 k_{2} \overline{\boldsymbol{\sigma}}(\mathbf{Q})}}{\bar{\sigma}(\mathbf{Q})} .
$$

Let $c=\min \left\{\frac{1}{2} \bar{\sigma}(\mathbf{Q})\|\mathbf{x}\|^{2}-k_{1} \epsilon\|\mathbf{x}\|-k_{2} \epsilon \mid \mathbf{x} \in \underline{E}\left(k_{0}\right) \backslash \underline{E}(\bar{k})\right\}$. From the argument in [18], one concludes the proof.

The above result indicates that the control resolution depends on the ellipsoid defined by $\eta(\epsilon)$. We can determine the values of $k_{1}$, $k_{2}$ to satisfy (16). It then becomes standard practice to choose the appropriate values of $\epsilon$ and $\bar{\sigma}(\mathbf{Q})$ to produce a small ellipsoid $E(\bar{k})$. The desired performance will then be satisfied in terms of steadystate error.

In the following, we will demonstrate the design procedure in terms of how to adjust the control parameters for the desired performance. We adjust the system performance by varying the poles of $\mathbf{A}_{\mathbf{K}}$. According to our theory, the only restriction on $\mathbf{A}_{\mathbf{K}}$ is that it be Hurwitz. Here we place all three poles at $-r \mathrm{rad} / \mathrm{sec}$. This choice allows us to derive closed-form solutions for $\mathbf{K}$ and $\mathbf{P}$, and thus makes it easy for demonstration purpose. Following this design, the value $r$ becomes the tuning parameter for the system performance. Increasing $r$ will speed up the response. It is also noted that this control drives the linear system without overshoot. Based on this design, the state feedback gain matrix becomes $\mathbf{K}=$ $\left[\left(-r^{3} / b\right)-\left(3 r^{2} / b\right)\right.$

$-(3 r-a / b)]^{T}$. The augmented state $x_{1}$ in (8) is not important, thus a Lyapunov function candidate can be chosen as

$$
\mathbf{Q}=\left[\begin{array}{ccc}
0 & 0 & 0 \\
0 & q_{22} & q_{23} \\
0 & q_{23} & q_{33}
\end{array}\right]
$$

Allow $q_{23}=\sqrt{q_{22} q_{23}}-\eta$ with $\eta>0$ so that $\left[\begin{array}{ll}q_{22} & q_{23} \\ q_{23} & q_{33}\end{array}\right]$ is a positive definite matrix. The choice for $q_{11}=q_{12}=q_{13}=$ $q_{21}=q_{31}=0$ only reflects the fact that $x_{1}$ is not important. With some algebra, the solution of $\mathbf{P}$ can be derived as

$$
\mathbf{P}=\left[\begin{array}{ccc}
\frac{r^{3} q_{33}+3 r q_{22}}{16} & \frac{3 r^{2} q_{33}+q_{22}}{16} & 0 \\
\frac{3 r^{2} q_{33}+q_{22}}{16} & \frac{3 r^{2} q_{33}+4 r q_{23}+3 q_{22}}{4 r} & \frac{r^{2} q_{33}+3 q_{22}}{16 r^{2}} \\
0 & \frac{r^{2} q_{33}+3 q_{22}}{16 r^{2}} & \frac{3 r^{2} q_{33}+q_{22}}{16 r^{3}}
\end{array}\right] .
$$




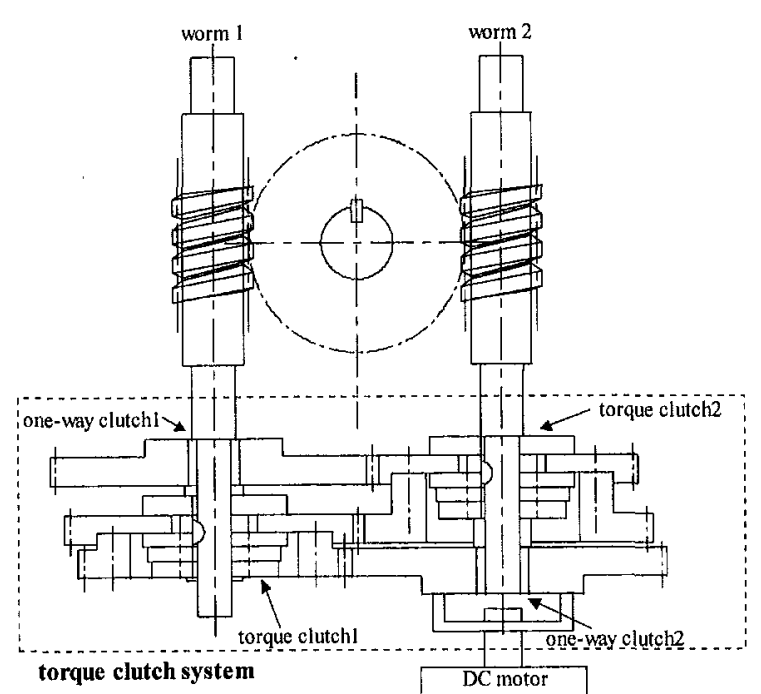

Fig. 2 Traction Type Drive Device (TTDD)

Designing the pole locations such that $\mathbf{P}$ is positive definite now becomes a straightforward approach.

The control effort $\phi(\mathbf{x})$ drives the system trajectories into an ellipse, thus it must be strong enough to suppress the uncertain term $d(\mathbf{x})$. This may be difficult in the case when position dependent friction exists. In this case the second term in (10) becomes important. If the knowledge for the friction characteristics is precise, $k_{1}$ and $k_{2}$ can be set to very small values to achieve very high positioning accuracy. Unfortunately, this is not easily brought about. There are three parameters to be determined in the proposed model. These parameters are the period, the amplitude and the phase of the force variation. The period depends on the mechanical design; therefore it can be determined precisely. The amplitude of the force variation depends on factors such as lubrication and load conditions. The fluctuation can be small under steady working conditions. Therefore, the uncertainty is also small and is easy to overcome. Notice that the integration action in our control law also serves to overcome the steady offset force. The situation is not quite the same for the phase variation. In most cases, the phase variation is almost as large as the period itself. Using a periodical compensation function can just make matters worse, because the compensation function may likely become out of phase with the friction. Phase variation may be the result of backlash, which leads to changes in the gear orientation. Certain phase adaptation scheme may become necessary in this case. This paper uses an anti-backlash mechanism to avoid the problems caused by phase variation.

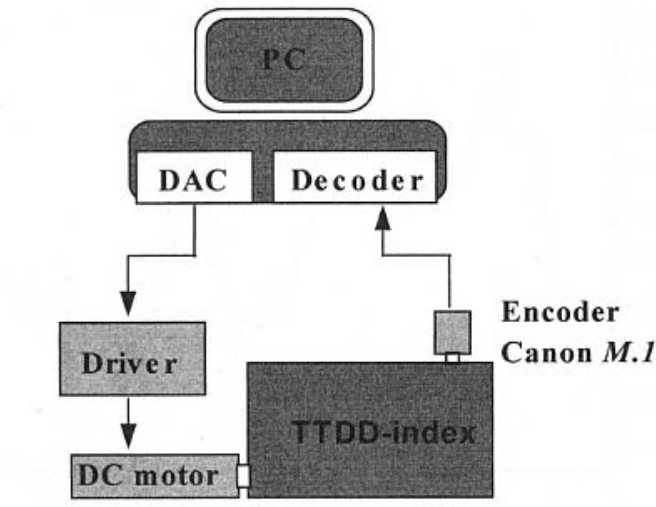

BALDOR SD45-20

Fig. 3 Experimental setup

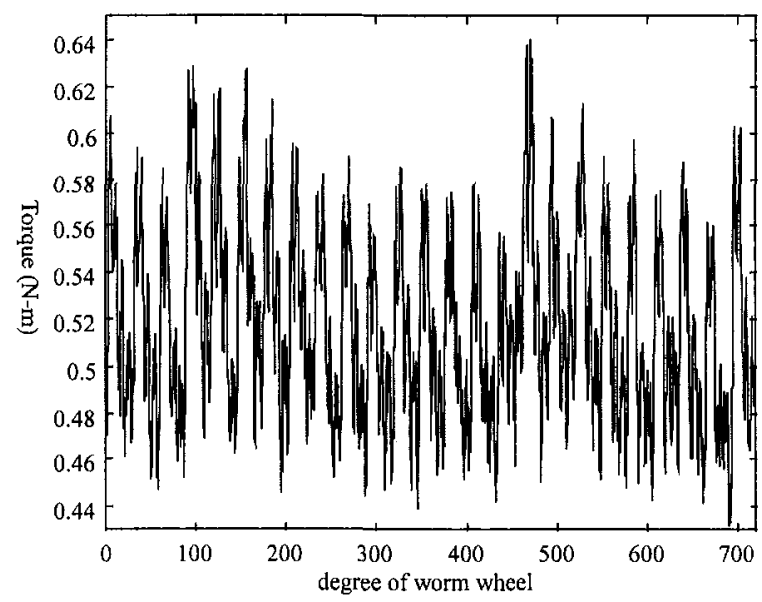

Fig. 4(a)

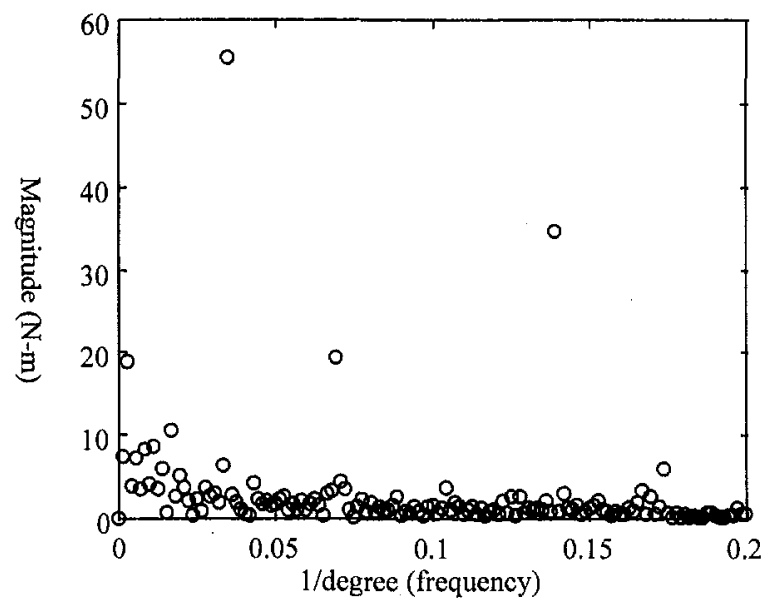

Fig. $4(b)$

Fig. 4 The stick position-dependent friction, (a) all of the stick friction characteristics, $(b)$ the amplitudes of friction in the angular frequency domain

3.1 Design Procedures. To summarize, designing the control parameters proceeds in three stages:

1. Design the linear feedback gains $\mathbf{K}$ appropriately so that the rise time of the system can be satisfied because this term is the dominant factor.

2. Select the values of $k_{1}$ and $k_{2}$ so that (16) is satisfied.

3. Select the values of $\mathbf{Q}$ subject to (21); then the controller in (10) can be derived.

Because position accuracy is of greatest concern in the index system, $q_{22}$ is selected such that it is greater than $q_{33}$.

\section{Experimental System}

The experiment was based on a Traction Type Drive Device (TTDD) [17]. To demonstrate the performance on the friction force variation, the experiment setup had to be free of the backlash effect. The TTDD as shown in Fig. 2 is an anti-backlash twin worm index system. The system is composed of two one-way clutches, two torque clutches and six gears to achieve backlashless and self-retaining operation. The TTDD index requires little concern about the mechanical backlash; however, friction becomes significant due to the use of torque clutches.

Figure 3 shows the experimental setup. The control PC reads the pulses from the encoder and sends the torque command to a BALDOR SD45-20-A1-A13 servomotor. The motor driver 
Table 1 Four dominant angular frequencies in the position-dependent stick friction

\begin{tabular}{lllll}
\hline & \multicolumn{1}{c}{1} & \multicolumn{1}{c}{2} & \multicolumn{1}{c}{3} & \multicolumn{1}{c}{4} \\
\hline frequency $(1 /$ degree $)$ & 0.0028 & 0.0347 & 0.0649 & 0.1389 \\
period (degree)(rad) & $360(6.283)$ & $28.8(0.503)$ & $14.4(0.251)$ & $7.2(0.126)$ \\
ampitude $(\mathrm{N} \cdot \mathrm{m})$ & 0.013293 & 0.039279 & 0.013689 & 0.024571 \\
phase (degree) & -135.9 & -109.2 & 178.7 & 168.5 \\
\hline
\end{tabular}

reaches saturation at $1.24 \mathrm{~N}$-m. A Canon M-1 super highresolution encoder is used for position sensing. The encoder produces $50,000 \mathrm{pulses} / \mathrm{rev}$ and achieves \pm 6.48 arc-second resolution. Unlike the common indexing system in which there is generally little concern on the position dependent friction behavior, the friction force in the TTDD index table displays strong positiondependent characteristics.

The first stage of the experiment was to study the friction characteristics. The stick friction forces were measured based on the smallest torque command that were able to move the worm wheel. Figure $4(a, b)$ presented the results. Figure $4(a)$ showed all of the frictional characteristics with respect to angular position. Figure $4(b)$ showed the amplitude variation in the spatial frequency domain. As indicated in this figure, the system displayed significant position-dependent friction characteristics. The angular position domain shown in Fig. 4(a) revealed that the maximum friction was almost twice as large as the minimum friction. The gear ratio of the TTDD was 12.5 . The worms and the worm wheel made contact at the same surface when the worms have rotated 25 turns and the worm wheels have rotated 2 turns. The stick forces were clearly combined with two separate periods. The short period came from the worm, and the period was $28.8^{\circ}\left(720^{\circ} / 25\right)$. The long

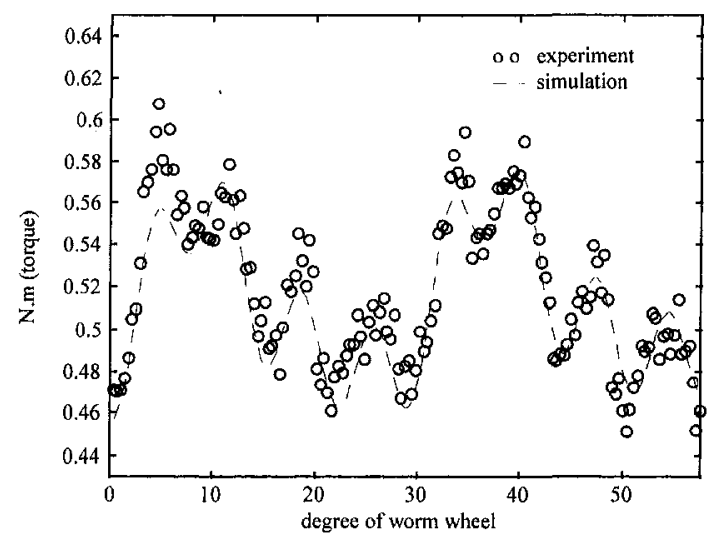

Fig. 5 Some of the experiment and simulation results for stick positiondependent friction

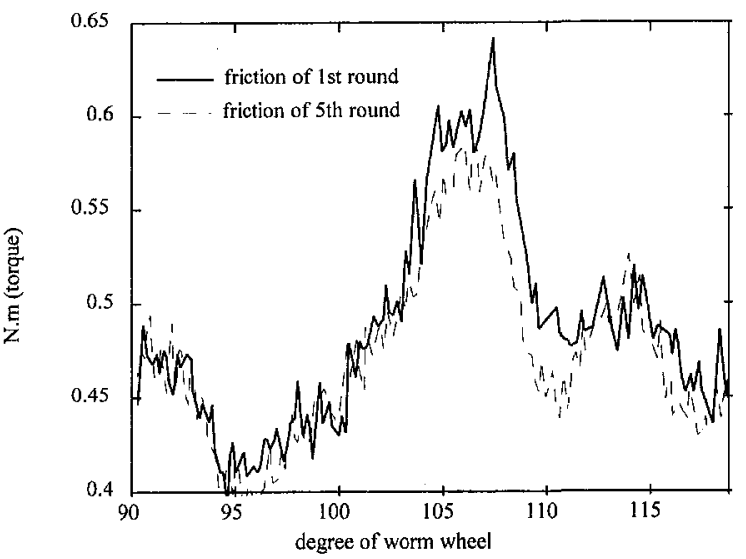

Fig. 6 The stick friction at the same contact surface under different runs period was produced by the worm wheel, and the period was $720^{\circ}$. The average force was $0.5172 \mathrm{~N}-\mathrm{m}$. Actually, the spatial frequency domain shown in Fig. $4(b)$ revealed that there were actually four frequency components. Table 1 listed their amplitudes, phases and periods. Combining the components, the position-dependent friction model was given by

$$
\begin{aligned}
& \tau_{\mathrm{pos}}(\theta)= 0.51720+0.013293 \cos (\theta-2.3725) \\
&+0.039279 \cos (12.5 \theta-1.9063)+0.013689 \cos (25 \theta \\
&\quad+3.1183)+0.024571 \cos (50 \theta+2.9402),
\end{aligned}
$$

where $\tau_{\text {pos }}$ represented the position-dependent stick friction and $\theta$ represented the angular position of the worm wheel (the unit of the $\theta$ was in rad.) The model in (23) captured the characteristics of the position-dependent stick friction. Figure 5 shown the comparison between the experimental and the simulation results. Figure 6 shown the position-dependency at the same contact surface under different trials. The amplitude variation was small and the system uncertainty caused by amplitude change was also small.

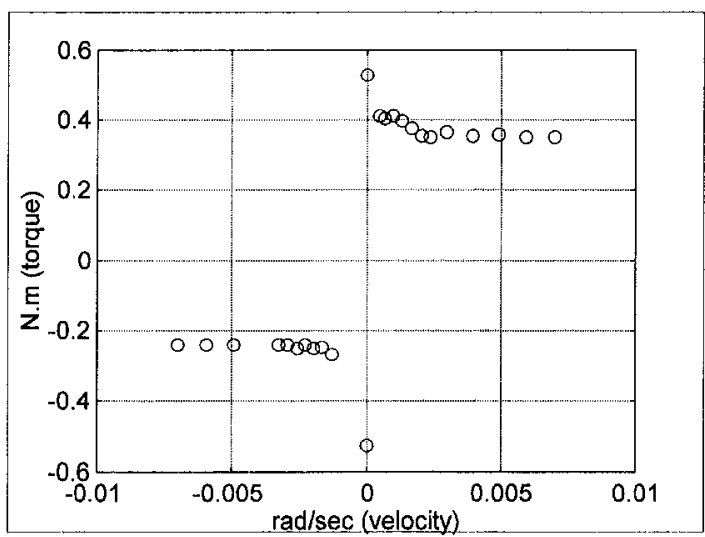

Fig. 7 Stick-slip and viscous friction

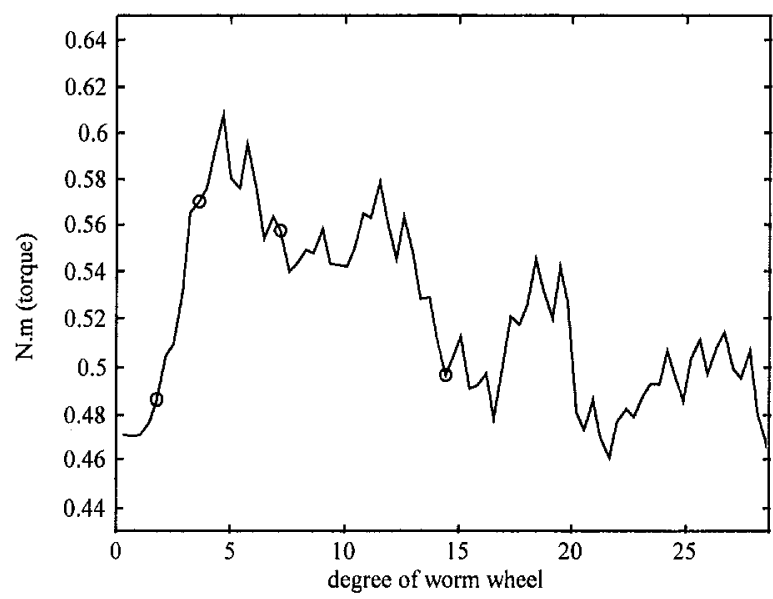

Fig. 8 Four different reference set-points for the experiments 
Table 2 Performance of different control laws compared for locations with different position dependent friction characteristics $\left(\right.$ error $=$ Pos $\left._{\text {comid }}-\theta\right)$

\begin{tabular}{|c|c|c|c|c|c|c|}
\hline \multirow{2}{*}{$\begin{array}{c}\text { Pos }_{\text {cand }}(\times 6.48 \\
\text { arc-sec })\end{array}$} & \multirow[b]{2}{*}{ Error $/ r$} & \multicolumn{3}{|c|}{ Linear control } & \multicolumn{2}{|c|}{ Proposed control } \\
\hline & & -15 & -20 & -30 & -15 & 20 \\
\hline \multirow{2}{*}{1000} & Mean & 1.625 & 0.375 & -0.625 & 0.250 & -0.125 \\
\hline & Stdev & 1.188 & 0.744 & 0.916 & 0.463 & 0.835 \\
\hline \multirow[t]{2}{*}{2000} & Mean & 2.250 & 0.500 & -1.875 & 0.125 & -0.250 \\
\hline & Stdev & 1.982 & 0.756 & 1.727 & 0.835 & 0.463 \\
\hline \multirow[t]{2}{*}{4000} & Mean & -1.625 & -2.750 & -3.625 & 0.250 & -0.875 \\
\hline & Stdev & 1.847 & 1.982 & 0.518 & 0.463 & 0.641 \\
\hline \multirow{2}{*}{8000} & Mean & -11.125 & -8.125 & -1.125 & 0.125 & -1.500 \\
\hline & Stdev & 2.642 & 3.182 & 1.126 & 0.354 & 2.070 \\
\hline
\end{tabular}

In the second stage the stick-slip and viscous friction with respect to the angular velocity were measured throughout their range of motion at constant velocities. The torque command required maintaining that velocity was recorded. The experiment was carried out at different velocities to generate the velocity dependency. The slope of the velocity relationship determined the viscous friction. By separating the velocity dependency and the spatial dependency, we were able to achieve a more reasonable position dependent friction model. Figure 7 presented the measurement results. The Strike-beck effect was clearly observed where there was a decreasing friction with increasing velocity around the low velocity region. This effect is sometimes the cause of system instability at low velocities. As indicated in Fig. 7, the viscous friction could be neglected in practice owing to a relative constant friction in the high velocity region.

\section{Experimental Results}

By matching the experiment results, the system inertia was calculated to be $1.454 \times 10^{-7} \mathrm{~m}^{2}-\mathrm{Kg}$, and the parameters in Eq. (1) were $a=0$ and $b=6.877 \times 10^{6}$. The stick position-dependent friction model $\tau_{\text {pas }}$ was given by (23). As indicated in Fig. 7, the parameters in the modified Karnopp model (4) were $\tau_{d}^{+}\left(\nu_{r}\right)=$ 0.67 for the slip friction in the counterclockwise direction and $\tau_{d}^{-}\left(\nu_{r}\right)=0.47$ for the slip friction in the clockwise direction. The zero region for $\sigma$ equaled $3.1 \times 10^{-5} \mathrm{rad} / \mathrm{s}$.

Consider the frictional system in (1) with a linear state feedback control $\left(u=\mathbf{K x}+\operatorname{pos}_{\text {cond }}\right)$ and with all three poles placed at $-r$. Assume that the initial conditions were $x_{1}(0)=0, x_{2}(0)=0$, and $x_{3}(0)=0$, and that $\operatorname{pos}_{\text {comi }}$ was positive and sufficiently great. If $\tau_{f}$ was a constant disturbance during the experiment, then the trajectories should be without overshoot, and $x_{2}(t)$ should stop directly on the desired point. The reason is obvious since there

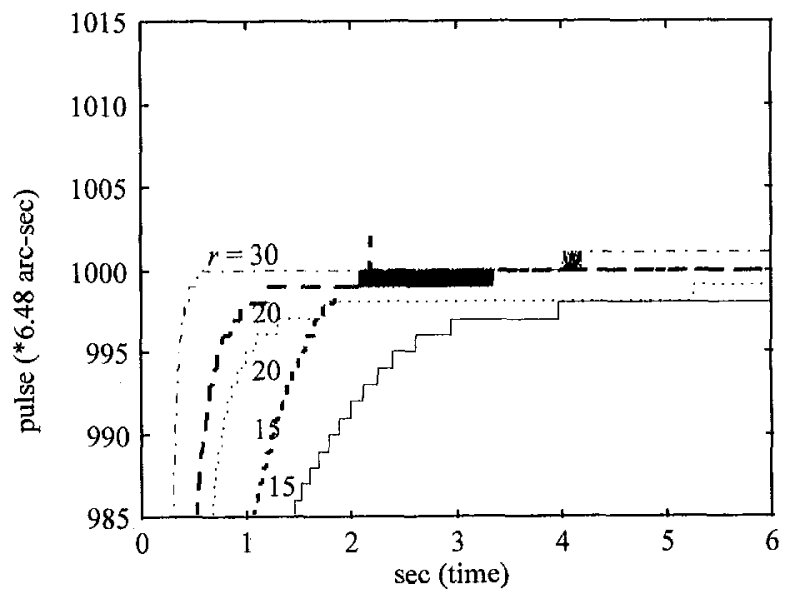

Fig. 9 Experimental results for increasing friction force region (position command $=1000$ pulses) were only first order closed-loop poles. If the reference point located at a location where $\tau_{f}$ was increasing or decreasing, the response would be very different. To investigate the phenomena, we selected four different locations for the target points. The commands for these positions are 1000, 2000, 4000, and 8000 encoder pulses. As shown in Fig. 8, the 1st and 2nd points located in regions with increasing friction, and the others are located in regions with decreasing friction. The experiment was then performed using linear controls with different pole assignment $(r=$ $15,20,30$ ). Table 2 summarized the mean and standard deviation

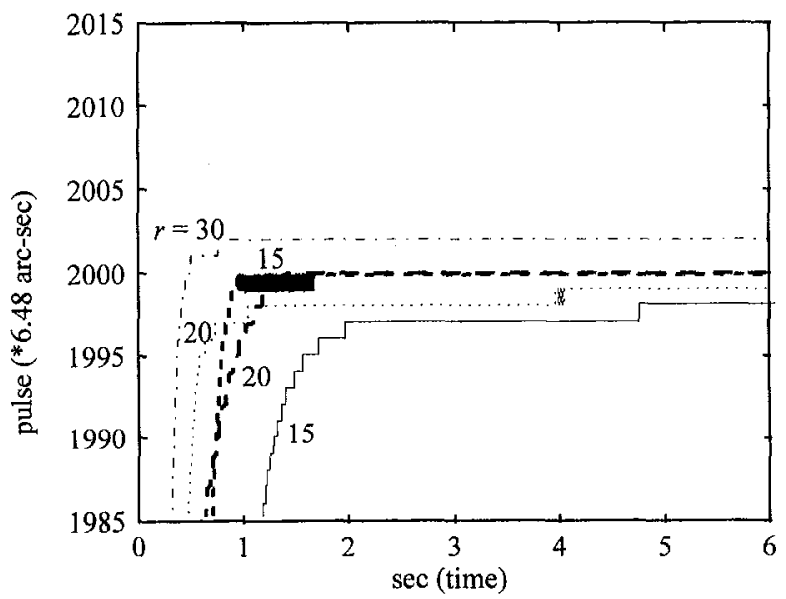

Fig. 10 Experimental results for increasing friction force region (position command $=2000$ pulses)

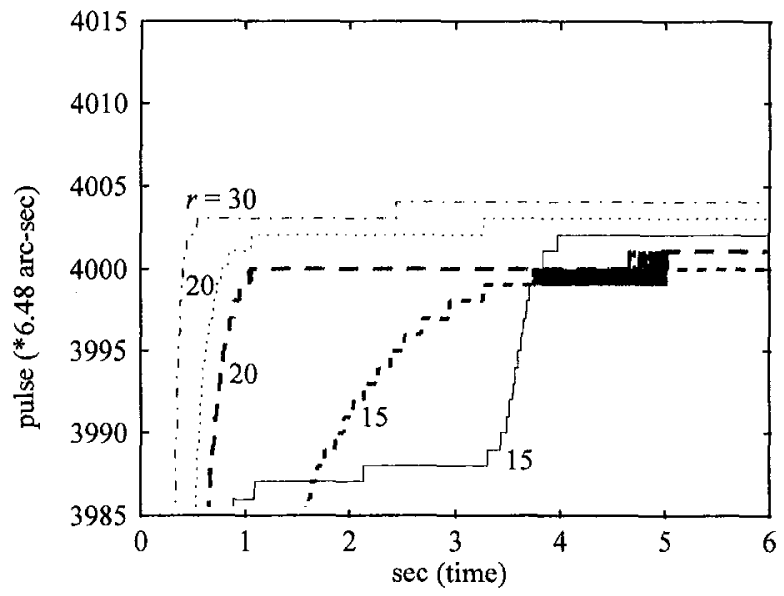

Fig. 11 Experimental results for decreasing friction force region (position command $=\mathbf{4 0 0 0}$ pulses) 


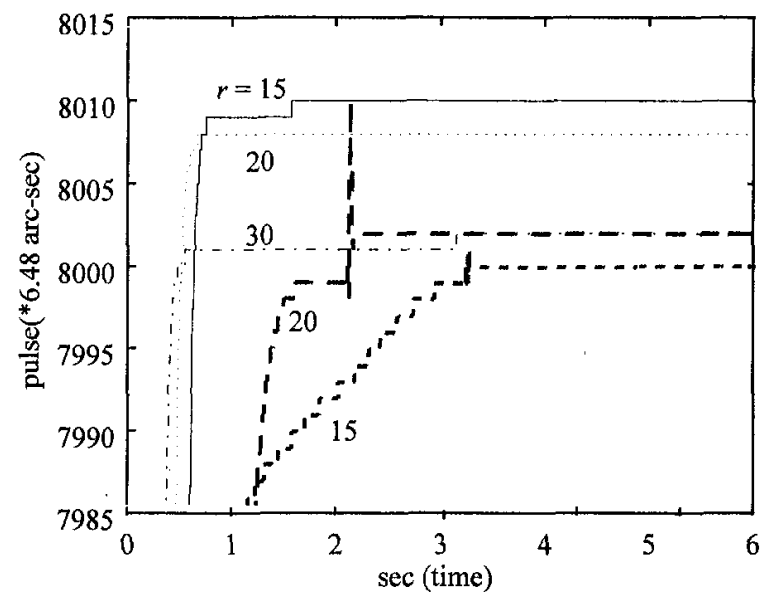

Fig. 12 Experimental results for decreasing friction force reglon (position command $=8000$ pulses)

of the steady-state errors under the different control laws and different target reference points. The Error in the table stood for $\operatorname{pos}_{\text {comd }}-\theta$. The positive error implied that the trajectory stopped in front of the desired point, and the negative error indicated that overshoot had occurred. The thin lines, solid and broken, in Figs. 9-12 depicted the experimental responses. Figures 9 and 10 showed the situations when the target points were located in regions where friction force increased with position, and Figs. 11 and 12 depicted the situations with decreasing friction force. Examining the result for the case with the poles placed at -15 , the mean of the error was found to be positive for target located in regions with increased friction, and negative for cases with decreased friction. For the case with increased friction, $x_{2}(t)$ stopped before it was able to reach the desired point because the actual friction force exceeded its anticipated value. Overshoot occurred in the other cases because the control exerted too much effort than actually required by the decreased friction. Furthermore, from the 2nd and 3rd columns in Table 2, increased control gains tended to reduce the control error. In summary:

1. If the target position is located in the region of increasing $\tau_{f}$, then the trajectories of $x_{2}(t)$ would stop in the front of the desired point; if $\tau_{f}$ is decreasing, then overshoot would occur.

2. Increasing control gains could enhance friction force rejection.

3. It is very difficult to obtain suitable linear feedback control gains that would be suitable for both the regions with increased and decreased friction force.

For the controller proposed in Eq. (10), we select $r=15$ and $r=20$ for the closed-loop poles, and $q_{22}=6.366 \times 10^{4}$, $q_{23}=2 \times 10^{3}, q_{33}=127.3, k_{1}=0.1, k_{2}=0.05$ and $\epsilon=$ $1 \times 10^{-4}$ for the controller calculation. The case with $r=30$ induced saturation on the motor driver and thus was not selected. The mean and standard deviation of the control errors were shown in the 4 th and 5 th columns of Table 2 ; the errors were obviously smaller with the proposed controller. The thick lines in Figs. 9, 10, 11 and 12 summarized the results for the control performances. As before, Figs. 9 and 10 showed the situation when the target point located in regions where friction force increased with increased angular displacement and for Figs. 11 and 12 the decreased friction force. Notably, the "ticking" behavior is the result of limited encoder resolution. As was found in Figs. 9-12, the performance of the system with the proposed nonlinear controller (10) was significantly improved. Regardless of whether the friction force was increasing or decreasing with position, the errors were smaller than 1 pulse, and no overshoot occurred in most cases. The result was expected because the effect of the decreased friction with increased position was compensated by the second term in (10). Moreover, the remainder of the uncertainty was overcome by the first and third terms. It is also noted in Fig. 12 that using the nonlinear control with high gain might cause saturation.

\section{Conclusions}

This paper proposed a new compensator design for systems with position dependent friction. The paper first presented a simplified friction model that describes the position dependent characteristics of the friction force. This model then gave rise to the design of a different nonlinear controller that would suppress the position dependent friction effect. It was shown that the proposed control achieved ultimate boundedness on the position dependent friction system even when frictional uncertainties existed. Based on the analysis, this paper also proposed a design procedure to achieve the desired control performance. Experimental results demonstrated that the new compensator maintained constant performance under different conditions for the position dependent friction force variation.

\section{Acknowledgments}

This work was supported by the National Science Council, Taiwan, under project number NSC84-2212-E-002-029.

\section{References}

1 Armstrong, B., 1988, "Friction: Experimental Determination, Modeling and Compensation," Proceedings of the 1988 IEEE International Conference on Robotics and Automation, Vol. 3, pp. 1422-1427.

2 Canudas, C., 1993, "Robust Control for Servo-Mechanisms Under Inexact Friction Compensation," Automatica, Vol. 29, No. 3, pp. 757-761.

3 Southward, S. C., Radeliffe, C. J., and MacCluer, C. T., 1991, "Robust Nonlinear Stick-Slip Friction Compensation," ASME Journal of Dynamic Systems, Measurement, and Control, Vol. 113, Dec., pp. 639-645.

4 Mentzeloplou, S. E., and Friedland, B., 1994, "Experimental Evaluation of Friction Estimation and Compensation Techniques," 1994 American Control Conference, Baltimore, MD, Vol. 3, pp. 3132-3136.

5 Phillips, Stephen M., and Ballou, Kevin R., 1993, "Friction Modeling and Compensation for an Industrial Robot," Journal of Robotic Systems, Vol. 10(7), pp. 947-971.

6 Dahl, P., 1968, "A Solid Friction Model," Tech. Rep., Aerospace Corp., EI Segundo, CA, Tor-01.58(3107-18)-1, 1968.

7 Armstrong, B., 1991, Control of Machines with Friction, Kluwer Academic Publishers, 1991.

8 Karnopp, D., 1985, "Computer Simulation of Stick-Slip Friction in Mechanical Dynamic Systems," ASME Journal of Dynamic Systems, Measurement, and CONTROL, Vol. 107, Mar., pp. 100-103.

9 Haessig, D. A., Jr., and Friedland, D. A., 1991, "On the Modeling and Simulation of Friction," ASME JouRnal of Dynamic Systems, MEAsurement, and CONTROL, Vol."113, Sept,, pp. 354-362.

10 Canudas, C., Olsson, H., and Lischinsky, P., 1995, "A New Model for Control of Systems with Friction," IEEE Transactions on Automatic Control, Vol. 40, No. 3 , Mar., pp. $419-425$.

11 Garofalo, F., and Leitmann, G., 1989, "Guaranteeing Ultimate Boundedness and Exponential Rate of Convergence for a Nominally Linear Uncertain Systems," aSme Journal of Dynamic Systems, Measurement, and Control, Vol. 111 , Dec., pp. $584-588$.

12 Huang, S. J., Yen, J. Y., and Lu, S. S., 1997, "Stability of PDF Controller with Stick-Slip Friction Drive Device," ASME Journal of Dynamic SYstems, MEasureMENT, AND CONTROL, Vol. 119, Sept., pp. 486 490, 1997.

13 Yang, Y. P., and Chu, J. S., 1993, "Adaptive Velocity Control of DC. Motor with Coulomb Friction Identification," ASME Journal of Dynamic Systems, MEASUREMENT, AND CONTROL, Vol. 115, Mar., pp. 95-102.

14 Yang, S., and Tomizuka, M., 1988, "Adaptive Pulse Width Control for Precise Positioning \-Under the Influence of Stiction and Coulomb Friction," asme Journal of Dynamic Systems, Measurement, and Control, Vol. 110 , Sept., pp. 221-227.

15 Canudas, C., Astrom, K. J., and Braun, K, 1986, "Adaptive Friction Compensation in DC Motor Drives," IEEE Trans. Rob. Auto., Vol. RA-3, No, 6, Dec., pp. $681-685$.

16 Phelan, R. M., 1987, Feedback Control Systems, (Privately Published Notes), Sibley School of Mechanical and Aerospace Engineering, Cornell University Press, Ithaca, NY.

17 Yang, T. H., 1993, "Double Acting Type Dynamic Back Spacing Removed Driving System," U.S. patent, No. 5265488.

18 Corless, M. J., and Leitmann, G., 1981, "Continuous State Feedback Guaranteeing Uniform Ultimate Boundedness for Uncertain Dynamic Systems," IEEE Transactions on Automatic Control, Vol. AC-26, No. 5, Oct., pp. 1139-1144. 